\title{
Study of hypoglycemia in neonates with low birth weight
}

\author{
Acharya E. ${ }^{*}$ \\ DOI: https://doi.org/10.17511/ijpr.2020.i08.03
}

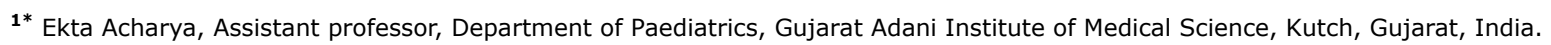

Background and Aim: The maintenance of normoglycemia in newborns depends upon the adequacy of glycogen stores, maturation of glycogenolytic and gluconeogenic pathways, and integrated endocrine response. The current study, therefore, proposes to observe the clinical profile of low-birth-weight babies concerning the occurrence of hypoglycemia. Materials and Methods: The neonates with a weight of fewer than 2500 grams were included in the study. A total of 100 infants were included in the study. They were divided into two groups with infants with hypoglycemia in one group and infants with normoglycemic in another group. The aseptic condition was maintained and with the help of heel prick, the capillary blood was collected. The capillary blood was screened with the help of reagent strips. Results: Out of the total 100 neonates, a total of 76 neonates were normoglycemic and 24 neonates had hypoglycaemic episodes. Overall 22 episodes of hypoglycemia were recorded. There was no significant association between birth weight and episodes of hypoglycemia. The majority of hypoglycaemic infants were male. Conclusion: Hypoglycemia associated with abnormal clinical signs (symptomatic hypoglycemia) has a poor shortand long-term outcome but evidence of risk in the absence of clinical signs (asymptomatic hypoglycemia) is inconclusive. Small for gestational age is a significant determinant for hypoglycemia. Hypoglycaemic episodes were significantly noticed in the first 24 hours as compared to another time interval.

Keywords: Hypoglycaemia, Low Birth Weight, Infants, Neonates, Episodes

Corresponding Author

Ekta Acharya, Assistant professor, Department of Paediatrics, Gujarat Adani Institute of Medical Science, Kutch, Gujarat, India.

Email: drektathacker@gmail.com
How to Cite this Article

To Browse
Acharya E. Study of hypoglycemia in neonates with low birth weight. Pediatric Rev Int J Pediatr Res. 2020;7(8):409-413.

Available From

https://pediatrics.medresearch.in/index.php/ijpr/arti cle/view/636

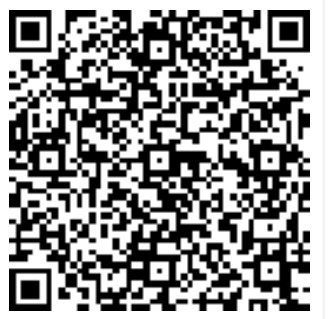

Manuscript Received 2020-11-07

Conflict of Interest No

Review Round 1
2020-11-17
Funding
Nil
2020 by Ekta Acharya and Published by Siddharth Health Research and Social Welfare Society. This is an Open Access article
licensed under a Creative Commons Attribution 4.0 International License https://creativecommons.org/licenses/by/4.0/ unported [CC BY 4.0].

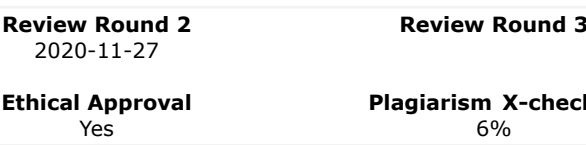

Review Round 3 2020-12-09

Note 


\section{Introduction}

World health organization has defined low birth weight as when the birth weight of an infant is 2499 $\mathrm{gm}$ or less. The weight is not respective of gestational age. In the long term, it has been known that infants born with a low birth weight (BW) for their gestational age are at risk of hypoglycemia even though its definition remains controversial [1]. A few studies have suggested that persistent glucose levels of less than $2.6 \mathrm{mmol} / \mathrm{L}$ in small-forgestational-age (SGA) preterm newborns may have long-term effects on their neurodevelopment. Hypoglycemia meaning a decreased level of sugar in the blood was coined by Harris in the late 19th century $[2,3]$. Neonatal hypoglycemia is a common metabolic disease due to the inability to maintain glucose homeostasis. The overall prevalence depends on birth weight, gestational age, and intrauterine growth retardation [4]. Since quite a proportion of neonates do not manifest any symptoms despite the existence of hypoglycemia, the exact incidence is difficult to work out. Undiagnosed hypoglycemia can have long-term neurological consequences; thus the emphasis is on prevention and early detection along with treatment of asymptomatic hypoglycemia [5]. Neonates, accounting for approximately $70 \%$ of all preterm births and about $8 \%$ of total births. Infants who are late preterm may appear mature, but they are physiologically, metabolically, and neurologically immature [6]. These infants are at higher risk for several problems including poor feeding, hypoglycemia, hypocalcemia, jaundice, infections, respiratory distress, failure to thrive, and hospital readmission [7]. Low birth weight babies have a high risk of developing hypoglycemia, hypocalcemia, acidosis hypoxia, and hypoproteinaemia. The clinical problems and outcomes of small for gestational age babies are very difficult as compared to preterm babies [8]. Symptomatic hypoglycemia, birth asphyxia, polycythemia, congenital malformations, and pulmonary hemorrhage is more common in term small for gestational age babies as compared to preterm small for gestational age babies [9]. Other problems like hyaline membrane disease, apnoeic attacks, inability to suck and swallow, aspiration of feeds, junctional obstruction, enterocolitis, hypothermia, hyperbilirubinemia, susceptibility to infections and intraventricular hemorrhage is more common in preterm small for gestational age babies as compared to small for gestational age babies.
Neonatal hypoglycemia is a common metabolic disease due to the inability to maintain glucose homeostasis. The overall prevalence depends on birth weight, gestational age, and intrauterine growth retardation. Since quite a proportion of neonates do not manifest any symptoms despite the existence of hypoglycemia, the exact incidence is difficult to work out $[4,10]$. In other words, symptomatic hypoglycemia is not as common as chemical hypoglycemia. The maintenance of normoglycemia in newborns depends upon the adequacy of glycogen stores, maturation of glycogenolytic and gluconeogenic pathways, and integrated endocrine response. The current study, therefore, proposes to observe the clinical profile of low birth weight babies concerning the occurrence of hypoglycemia.

\section{Materials and Methods}

The present study was done in the pediatric department of the medical hospital and the associated medical college. The present is the hospital-based prospective longitudinal study. The ethical committee of the institute was informed about the study and the ethical clearance certificate was obtained from them before the start of the study. All the parents were informed about the study and the written informed consent was obtained from them before the start of the study.

The neonates with a weight of fewer than 2500 grams were included in the study. Whereas the mother of the neonates diagnosed with diabetes, birth asphyxia, presence of endocrine deficiencies, and the presence of congenital malformations was excluded from the study. A total of 100 infants were included in the study. They were divided into two groups with infants with hypoglycemia in one group and infants with normoglycemic in another group.

Lethargy, jitteriness and seizures, tremor, apnea, poor feeding, etc were considered to be clinical signs of hypoglycemia if they were unexplained by other diagnoses and corrected with the provision of glucose. Infants were considered asymptomatic if low plasma glucose concentration was not associated with clinical signs. All neonates were weighed at birth with an electronic weighing machine with an accuracy of $\pm 5 \mathrm{~g}$. The gestational assessment was done by the new Ballard score. Neonates were managed as per the standard protocol. Asymptomatic hypoglycemia was first treated by adjusting the enteral feeding regimen. 
If this approach failed, intravenous therapy was instituted. For the collection of the sample, the aseptic condition was maintained, and with the help of heel prick, the capillary blood was collected. The venous blood was also collected. The capillary blood was screened with the help of reagent strips. The venous blood was also sent for a detailed laboratory investigation. The autoanalyzer was used for the investigation of the venous glucose level. For the statistical analysis, the data were presented in the form of statistical tables and charts. SPSS software version 20 was used for the statistical analysis of the study.

\section{Results}

The present study was done to study the clinical profile of low birth weight babies concerning the occurrence of hypoglycemia. A total of 100 neonates were included in the study. The age of the mother of the neonates included in the study was of age ranged from 24 to 34 years. The mean age of the mother was $29 \pm 5$ years. A total of 38 mothers age ranges of 24 to 28 years, there were 36 mothers in the age range of 29 to 32 years and there were 26 women with the age of more than 33 years. The majority of the infants were born to the mother age range 24 - 28 years. There was no significant association between the age of the mother and hypoglycemia (Table 1 ).

A total of 66 neonates were given intravenous fluids at the time of birth. Intragastric feed was given to 4 neonates whereas in 34 neonates there was oral feed done. The majority of neonates were given intravenous fluids initially. When the difference between hypo and normoglycemic neonates has been assessed no statistical difference in respect to the method of feeding and episodes of hypoglycemia was found.

Out of the total 100 neonates, a total of 76 neonates were normoglycemic and 24 neonates had hypoglycaemic episodes. Overall 22 episodes of hypoglycemia were recorded. There was no significant association between birth weight and episodes of hypoglycemia. The majority of hypoglycaemic infants were male. There was no significant association between gender and episodes of hypoglycemia. The majority of hypoglycaemic infants were born with low birth weight. There was no significant association between birth weight and episodes of hypoglycemia. (Table 2).
A total of 24 neonates were found to be hypoglycaemic, of which there were 18 neonates with small gestational age and 6 neonates were of appropriate age. The difference was found to be statistically significant. Hypoglycemia was more common in small for gestational age infants. When the blood group was assessed in the infants, the majority of hypoglycemic infants had $\mathrm{O}+$ blood group. However, there was no significant association between baby blood group and episodes of hypoglycemia $(P=0.143)$.

Table-1: Distribution of study participants according to Age groups.

\begin{tabular}{|l|l|l|}
\hline \multicolumn{1}{|c|}{ Age of the mother (Years) } & Number & Percentage \\
\hline $24-28$ & 36 & 36 \\
\hline $29-32$ & 26 & 26 \\
\hline More than 32 years & 38 & 38 \\
\hline Total & 100 & 100 \\
\hline
\end{tabular}

Table-2: Distribution of Neonates according to Glycemic Status.

\begin{tabular}{|l|l|l|}
\hline \multicolumn{1}{|c|}{ Glycemic status of Neonates } & Number & Percentage \\
\hline Normoglycemic & 76 & 76 \\
\hline Hypoglycaemic & 24 & 24 \\
\hline Total & 100 & 100 \\
\hline
\end{tabular}

\section{Discussion}

Hypoglycemia essentially results from either decreased production or excessive utilization of glucose reserves. Thus, hypoglycemia occurs in a neonate who is born with low glycogen and fat stores with limited capacity to generate glucose via the gluconeogenesis pathway or excessive peripheral tissue utilization of glucose like in an infant of a mother with insulin-dependent diabetes [11]. Hypoglycemia associated with abnormal clinical signs (symptomatic hypoglycemia) has a poor short- and long-term outcome but evidence of risk in the absence of clinical signs (asymptomatic hypoglycemia) is inconclusive. The diagnosis and management of hypoglycemia depend mostly on the cause and severity of hypoglycemia, the clinical presentation, and the underlying etiology. Thus, the treatment plan should be individualized for each infant [12].

In our present study, the incidence of hypoglycemia in the low weight neonates was found to be $24 \%$.in the literature different studies have reported the different incidents. In the study done by Dias $\mathrm{E}$ and Gada S [13], they reported that the incidence of hypoglycemia found to be $17 \%$, and in the study done in Jonas D et al $\lceil 14\rceil$. 
In the present study, the weight of neonates ranged from $600 \mathrm{~g}$ to $2600 \mathrm{~g}$. The mean birth weight of neonates was found to $1900 \mathrm{~g}$. In the literature, various studies are reporting varying weight distribution. The study done by Budhathoki $\mathrm{S}$ et al [8] reported the mean weight to found to be 1600 g. The present study correlating with hypoglycemia depicted that out of 24hypoglycemic infants $58.3 \%$ were low birth weight, $41.7 \%$ were very low birth weight. But no significant association was found. ( $p-$ value $>0.05$ ). Similarly, Hawdon JM et al [15] stated the most common risk factor for hypoglycemia was low birth weight or borderline low birth weight.

When the distribution of the infants was done as per the hypoglycaemic status and also the gestational age; it showed that hypoglycaemic episodes are more common in the gestational age of 34 to 36 months. Narayan $\mathrm{S}$ et al studied that hypoglycemia was encountered in a variety of neonatal conditions among those prematurity was one. In the present study, out of 100 infants, the normal vaginal delivery was done in 56 infants and in 44 infants the delivery was done by lower segment cesarean section. In the study done by Dias E and Gada S [13], they concluded that mode of delivery is one of the determinants of hypoglycemia.

\section{Conclusion}

Small for gestational age is a significant determinant for hypoglycemia. Hypoglycaemic episodes were significantly noticed in the first 24 hours as compared to another time interval.

\section{What does the study adds to the existing knowledge?}

However, long-term prospective studies are needed to evaluate the future neurological damage in asymptomatic healthy term babies with hypoglycemia.

\section{Reference}

01. Cutland CL, Lackritz EM, Mallett-Moore $T$, Bardají A, Chandrasekaran R, Lahariya C, et al. Low birth weight- Case definition \& guidelines for data collection, analysis, and presentation of maternal immunization safety data. Vaccine. 2017;35(48Part A)6492-6500.

doi: $10.1016 /$ j.vaccine.2017.01.049 [Crossref]
02. Sharma A, Davis A, Shekhawat PS. Hypoglycemia in the preterm neonateetiopathogenesis, diagnosis, management and long-term outcomes. Transl Pediatr. 2017;6(4)335-348.

doi: $10.21037 /$ tp.2017.10.06 [Crossref]

03. Heinrich M. Ethnopharmacology and drug discovery, Comprehensive Natural Products II. Chemistry and Biology, Development \& Modification of Bioactivity. 2013;3;351-381. [Crossref]

04. De AK, Biswas R, Samanta M, Kundu CK. Study of blood glucose level in normal and low birth weight newborns and impact of early breast feeding in a tertiary care centre. Annals of Nigerian Medicine. 2011;5(2)53-58.

doi: $10.4103 / 0331-3131.92951$ [Crossref]

05. Barkovich AJ, Ali F, Rowley HA, Bass N. Imaging patterns of neonatal hypoglycemia. Am J Neuroradiol. 1998;19(3)523-528.

[Crossref]

06. Mally PV, Bailey S, Hendricks-Muñoz KD. Clinical issues in the management of late preterm infants. Curr Probl Pediatr Adolesc Health Care. 2010;40(9)218-233.

doi: 10.1016/j.cppeds.2010.07.005 [Crossref]

07. Purdy IB, Melwak MA. Who is at risk? High-risk infant follow-up. Newborn Infant Nursing Rev. 2012;12(4)221-226.

[Crossref]

08. Saini A, Gaur BK, Bhalla AS, Antil PK, Maini B, Bharadwaj AK. Clinical Profile of Low Birth Weight Neonates Admitted in NICU- A Hospital Based Study. J Med Sci Clin Res. 2019;7(1). doi: $10.18535 / j m s c r / v 7 i 1.67$ [Crossref]

09. Singh G, Chouhan R, Sidhu K. Maternal factors for low birth weight babies. Med J Armed Forces India. $2009 ; 65(1) 10-12$.

doi: $10.1016 /$ S0377-1237(09)80045-2 [Crossref]

10. Barbosa ADM, Júnior IF, de Lima GM. Neonatal Hypoglycemia. Selected Topics in Neonatal Care. 2018;63.

[Crossref] 
11. Sperling MA, Menon RK. Differential diagnosis and management of neonatal hypoglycemia. Pediatr Clin. 2004;51(3)703-723.

doi: $10.1016 / \mathrm{j} . \mathrm{pcl} .2004 .01 .014$ [Crossref]

12. Greeley SAW, Tucker SE, Naylor RN, Bell GI, Philipson LH. Neonatal diabetes mellitus: a model for personalized medicine. Trends Endocrinol Metabol. 2010;21(8)464-472. doi: 10.1016/j.tem.2010.03.004 [Crossref]

13. Dias E, Gada S. Glucose levels in newborns with special reference to hypoglycemia- a study from rural India. J Clin Neonatol. 2014;3(1)35-38. doi: $10.4103 / 2249-4847.128729$ [Crossref]
14. Jonas D, Dietz W, Simma B. Hypoglycemia in newborn infants at risk. Klinische Pädiatrie. $2014 ; 226(5) 287-91$.

doi: $10.1055 / \mathrm{s}-0034-1385928$ [Crossref]

15. Hawdon JM, Beer J, Sharp D, Upton M. Neonatal hypoglycaemia- learning from claims. Arch Dis Child Fet Neonatal Ed. 2017;102(2)F110-F5.

doi: 10.1136/archdischild-2016-310936 [Crossref] 